\title{
A reliable method of determining wound healing rate
}

\author{
$\begin{array}{lll}\text { D. Cukjati } & \text { S. Reberšek } & \text { D. Miklavčič }\end{array}$ \\ Faculty of Electrical Engineering, University of Ljubljana, Ljubljana, Slovenia
}

\begin{abstract}
Several wound healing rate measures have been introduced with the main goal of enabling quantification of the effects of various therapeutic modalities on the healing of open wounds. Different definitions of wound healing rate render comparison of clinical results difficult. The goal of the present study was to propose a measure of wound healing rate that is independent of initial wound extent and to present a method of wound healing rate prediction. Comparisons were made of wound healing rate defined as absolute area healed per day, percentage of initial area healed per day and advance of the wound margin towards the wound centre per day. Analysis was performed on 300 wound cases. A disadvantage of wound healing measures that either use absolute area healed per day or percentage of initial area healed per day is their very limited use for comparing healing rates of wounds with different initial sizes. This disadvantage was overcome by incorporating a wound perimeter; thus obtaining a measure of the advance of the wound margin towards the wound centre. A definition of healing rate expressed as the greatest average wound margin distance from the wound centre divided by the time to complete wound closure is proposed. Because not all wounds are closed in the observation period, the time to complete wound closure has to be predicted. A method of wound healing rate prediction is presented based on a delayed exponential model the parameters of which are obtained from at least five weekly wound area measurements. Paired $t$ tests between actual time needed to complete wound closure and the predicted time resulted in $p=0.062$ after four, 0.484 after five and 0.900 after six weeks of observation.
\end{abstract}

Keywords-Chronic wounds, Wound healing rate, Wound healing dynamics

Med. Biol. Eng. Comput., 2001, 39, 263-271

\section{Introduction}

A CUTANEOUS WOUND is any loss of skin integrity due to injury or illness. Since intact skin is of vital importance to protect the organism against the environment, regenerative mechanisms must be activated to resolve a defect. The primary goal of healing is to restore the preinjured form and function of the skin. Cutaneous would healing is a dynamic biological process that begins after tissue injury and is divided into three overlapping phases: inflammatory, proliferative phase and maturation (WALDORF and FEWKES, 1995). If any of these phases is suppressed, wound healing is prolonged or even prevented. Reasons for slower or retarded healing can be local, such as bacterial infection that prolongs the inflammatory phase; lower oxygen tension that prolongs the proliferative phase; or systemic, such as injuries of the nervous system, metabolic and ageing problems that affect one or more phases of wound healing. When conservative methods of wound care cannot facilitate wound healing, the wound is considered to be chronic. Such chronic wounds can last for months or even years, hinder the normal course of rehabilitation and represent a major social, medical and economic problem.

Correspondence should be addressed to Dr D. Cukjati; e-mail: david.cukjati@fe.uni-lj.si

First received 28 July 2000 and in final form 15 November 2000

MBEC online number: 20013556

(C) IFMBE: 2001
A review of the literature reveals that many treatment modalities have been employed and reported to facilitate chronic wound healing, including wound dressings (KANNON and GARETT, 1995), low energy laser (GOGIA, 1995a), ultrasound (BROWN, 1995), ultrasound/ultraviolet treatment (NeSSBAUM et al., 1994), hyperbaric oxygen (GoGIA, 1995b), skin substitutes (SINGER and CLARK, 1999; BREM et al., 2000), use of growth factors (MARTIN et al., 1992; KUNIMOTO, 1999) and electrical stimulation (VODOVNIK and KARBA, 1992).

One of the treatment modalities that is well documented and has been shown to facilitate chronic wound healing is electrical stimulation. Since WOLCOTT et al. (1969) published promising results of an extensive clinical study involving electrical stimulation treatment, many reports have confirmed additional positive effects of electrical stimulation, although different stimulation protocols were used. Practically all reports are rather exclusive regarding their methods for quantification of treatment results (Table 1). Due to the different quantification methods used it is impossible to make a quantitative analysis of the different wound measurement methods. The purpose of this paper is to present a wound measurement method that is suitable for application to different wound aetiologies, sizes and shapes.

Comparable and uniform quantification methods are important for determining an optimal treatment regime. This paper examines and compares different measurement methods used by various authors, and proposes a uniform method of assessing wound healing that accurately reflects wound healing rate. Evaluation of different wound measurement methods was based on the data of 300 chronic wounds that were primarily 


\begin{tabular}{|c|c|c|c|c|}
\hline Measured values & Wound healing rate definition & Units & Frequency of measurements & Reference \\
\hline width, length & $\frac{\pi / 4 \times \text { length }_{0} \times \text { width }_{0}-\pi / 4 \times \text { length }_{f} \times \text { width }_{f}}{\left(\text { day }_{f}-\text { day }_{0}\right) \times \pi / 4 \times \text { length }_{0} \times \text { width }_{0}} \times 100$ & $\% /$ day & initial and final & LYMAN et al., 1970 \\
\hline width, length & $\frac{\text { length }_{0} \times \text { widt }_{0}-\text { length }_{4} \times \text { width }_{4}}{4 \times \text { length }_{0} \times \text { width }_{0}} \times 100$ & $\% /$ week & five in four weeks & FEEDAR et al., 1991 \\
\hline area & $\frac{\text { area }_{0}-\text { area }_{i}}{\text { area }_{0}} \times 100 ; i=0,2,4,6,8,12$ & $\%$ & $\begin{array}{l}\text { initial and after } \\
2,4,6,8 \text { and } 12 \text { weeks }\end{array}$ & $\begin{array}{l}\text { LUNDEBERG } \text { et al., } \\
1992\end{array}$ \\
\hline area & Time needed to complete wound closure. & days & initial and final & BIRKE et al., 1992 \\
\hline area & $\begin{array}{l}\text { Fitting } S_{0} \exp (-\theta t) \text { or } S_{0}+(-A t) \text { to } \\
\frac{\text { area }_{i}}{\text { area }_{0}} \times 100 ; i=1,2, \ldots, n \\
\text { Parameters } \theta \text { or } A \text { were defined as wound healing rate. }\end{array}$ & $\% /$ day & $2<n \leqslant 5$ in four weeks & $\begin{array}{l}\text { JERČINOVIĆ et al., } \\
1994\end{array}$ \\
\hline $\begin{array}{l}\text { granulation, } \\
\text { infection, } \\
\text { drainage, } \\
\text { necrosis, eschar }\end{array}$ & $\begin{array}{l}\text { Average change in Sessing scale ( } 7 \text { stage classifi- } \\
\text { cation system) between two consecutive scoring. }\end{array}$ & scaling & twice per week & $\begin{array}{l}\text { FERRELL et al., } \\
1995\end{array}$ \\
\hline area, perimeter & $\frac{\text { area }_{0}-\text { area }_{2}}{\frac{1}{2}\left(\text { perimeter }_{0}+\text { perimeter }_{2}\right)\left(\text { day }_{2}-\text { day }_{0}\right)}$ & $\mathrm{mm} /$ day & initial and after two weeks & GORIN et al., 1996 \\
\hline area & $\frac{\text { area }_{0}-\text { area }_{4}}{\text { area }_{0}} \frac{28}{\text { day }_{4}-\text { day }_{0}} \times 100$ & $\begin{array}{l}\% / \text { four } \\
\text { weeks }\end{array}$ & initial and after four weeks & JOHNSON, 1997 \\
\hline area & 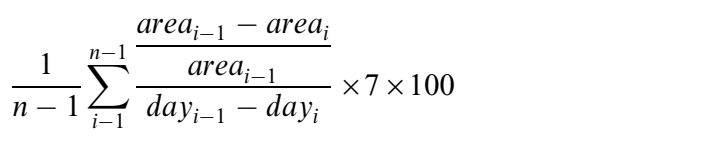 & $\% /$ week & weekly & $\begin{array}{l}\text { BAKER et al., } \\
1997\end{array}$ \\
\hline $\begin{array}{l}\text { area, exudate, } \\
\text { appearance }\end{array}$ & $\begin{array}{l}\text { Linear regression to PUSH (Pressure Ulcer Scale } \\
\text { for Healing) values in week } 0,2,4,6 \text { and } 8 .\end{array}$ & scaling & $\begin{array}{l}\text { initial and after } \\
2,4,6 \text { and } 8 \text { weeks }\end{array}$ & $\begin{array}{l}\text { CUDDIGAN, 1997, } \\
\text { BARTOLUCCI and } \\
\text { THOMAS, } 1997\end{array}$ \\
\hline
\end{tabular}

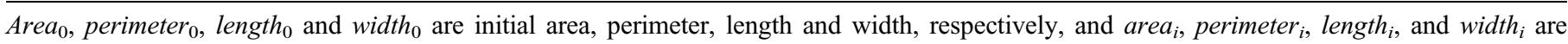
wound area, perimeter, length and width after $i$ weeks, respectively. Day $y_{0}$ is initial assessment date and $d a y_{i}$ is assessment date after $i$ weeks and $d a y_{f}$ is final assessment date. $n$ is number of sequential wound area measurements.

evaluated for the purpose of studying the effects of electrical wound treatment (STEFANOVSKA et al., 1993; JERČINOVIĆ et al., 1994; KARBA et al., 1997). The study included wounds of various aetiologies (e.g. vascular ulcerations, amputation wounds, pressure ulcers, neuropathic ulcerations), locations and different treatments in patients with different primary diagnoses (e.g. spinal cord injury, diabetes mellitus, sclerosis multiplex, vascular diseases). The wound healing rate measure should describe the healing process irrespective of wound aetiology, location and treatment.

\section{Wound status assessment}

Assessment of wound status is essential in clinical trials and practice for monitoring treatment efficacy. LAZARUS et al. (1994) proposed guidelines for the assessment of wounds. They listed attributes that are clues to the cause, pathophysiology and status of the wound. Assessment of wound status should begin with the extent of the wound. Because the extent of the wound changes with time, it requires periodic assessment. There are several techniques that may be employed to assess wound extent. To be clinically acceptable, the assessment of chronic wound healing has to be noninvasive, inexpensive and practical enough to be regularly used by clinicians. As presented in Table 1, most often wound area, wound perimeter or mutually perpendicular diameters (largest diameter of the wound and diameter taken at right angle to the largest one) are assessed. Wound volume and depth assessment techniques are invasive (dental moulds) (COVINGTON et al., 1989) or require expensive equipment (stereoscopy, MRI) (PLASSMANN and JONES, 1992) and are rarely used. Since wounds are often irregular in shape and heal asymmetrically, different estimates of wound area are used. Acetate tracings can provide the most accurate description of wound area and perimeter but require manual or computer planimetry. Estimates of wound area can be calculated from the product of two mutually perpendicular perimeters, or by calculation of the area of a circle or ellipse from measured diameters. Surface area can also be estimated by simply comparing ulcers to pre-drawn circles or ellipses of known area. STEFANOVSKA et al. (1993) have established that the approximation of the wound area by the area of the ellipse, calculated from two diameters, does not significantly differ from the area obtained by planimetry. The measurement of two diameters is simple, reproducible, and easy to perform at the bedside.

Another wound status assessment possibility are scaling systems. They are based on an assumption that wound extent is not sufficiently descriptive. Besides wound extent they also incorporate a description of necrosis, surrounding skin colour, peripheral tissue edema and induration, granulation tissue, epithelialization, infection, drainage, eschar and exudates. Drawbacks of such scaling systems are their reliability and complexity. The most widely used pressure ulcer scaling system is the four-stage system developed by the National Pressure Ulcer Advisory Panel (NPUAP) (NATIONAL PRESSURE UlCER ADVISORY PANEL (NPUAP), 1989). NPUAP also warns that staging should not be used to determine progress towards wound healing, because a stage IV pressure sore is always a stage IV ulcer no matter how it is healing. For this reason several tools have been proposed, that are responsive to changes during wound healing. These systems are still in various stages of testing, but two of the most promising 
appear to be the seven-point categorical Sessing scale (FERRELL et al., 1995) and PUSH (BARTOLUCCI and THOMAS, 1997) which is based on area, exudates and wound appearance. Scaling systems are widely used as a wound assessment alternative, as they are practical for daily monitoring. However, it is still not clear if it is appropriate to use them for the follow-up of changes in wound healing. The small number of stages makes them easy to use but at the same time makes them insufficiently sensitive for wound healing progress description. Bases on the above considerations wound extent should be evaluated for monitoring wound status when progress towards wound healing has to be determined.

\section{Wound healing process dynamics}

If the assessment of wound extent is a one-dimensional quantitative value (a scalar) and is periodically assessed, linear or nonlinear regression can reveal wound healing dynamics over time. Thus our measurement method evaluates wound extent by including perimeter, area or two mutually perpendicular diameters of the wound. One-dimensional wound extent measurement can be determined by using any one of these variables or any of their combinations.

GILMAN (1990) defined a measure of wound extent that incorporates wound area and perimeter. It was termed the advance of the wound margin toward the wound centre (eqn 1):

$$
d_{i}=\frac{\Delta S_{i}}{\bar{p}_{i}}
$$

where index $i$ describes the wound extent assessment at time $t_{i}, t_{0}=0$ is the time of the initial wound assessment. $\bar{p}_{i}=\left(p_{0}+p_{i}\right) / 2[\mathrm{~mm}]$ is mean wound perimeter calculated from initial perimeter and wound perimeter at time $t_{i}>t_{0}$, and $\Delta S_{i}=S_{0}-S_{i}$ is absolute change in wound area $\left[\mathrm{mm}^{2}\right]$ in time interval $t_{0}$ to $t_{i}$. The advantage of this measure is its independence of initial wound extent. In spite of this, the measure is rarely used.

The majority of researchers use measures of wound extent that incorporate only wound area calculated from two diagonals or using planimetry. Wound extent is defined either as absolute or normalised wound area.

Before defining wound healing rate based on one-dimensional wound extent measurements, the dynamic of the healing process should be examined over time. Dynamic of the absolute and normalised wound area over time is similar, therefore we have concentrated on the normalised wound area, where wound area is divided by the initial wound area and multiplied by 100 . It has been assumed that the wound can be estimated with an ellipse and the wound area $S_{i}$ calculated from mutually perpendicular wound diameters $a_{i}$ and $b_{i}$ measured at time $t_{i}$ (eqn 2) according to STEFANOVSKA et al. (1993) is:

$$
S_{i}=\frac{\pi}{4} a_{i} \cdot b_{i} \quad i=0,1, \ldots, n-1
$$

where index $i$ describes wound extent assessment at time $t_{i}, t_{0}=0$ is time of initial wound assessment and $n$ is the number of periodic assessments. Diameters $a_{i}$ and $b_{i}$ define the width to length ratio $r_{i}$ (eqn 3 ), which describes wound shape $\left(r_{i} \leqslant 1\right)$.

$$
r_{i}=\frac{b_{i}}{a_{i}}
$$

Wound perimeter, estimated with the ellipse at rime $t_{i}$, was calculated according to:

$$
p_{i}=\pi\left[\frac{3}{4}\left(a_{i}+b_{i}\right)-\frac{1}{2} \sqrt{a_{i} \cdot b_{i}}\right]
$$

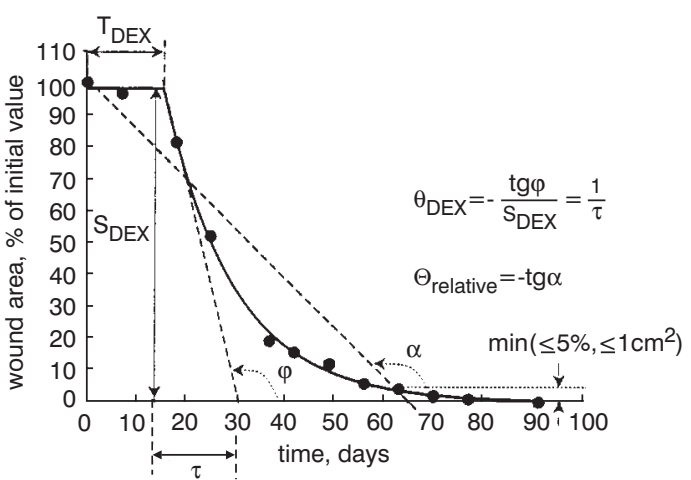

Fig. 1 Example of following wound area and application of the delayed exponential model to normalised data. $S_{D E X}, \theta_{D E X}, T_{D E X}$ are the calculated parameters of the delayed exponential model and $\Theta_{\text {relative }}$ a measure of wound healing rate. (-) Fitted delayed exponential model; (•) normalised wound area

Determining the normalised wound area or the advance of the wound margin towards the wound centre over time can provide a general overview of wound healing dynamics. In the following section both measures are considered.

In a recent study (CUKJATI et al., 1998; 2000) it was established that a decrease (or increase) in normalised wound area over time is best described by a delayed exponential model, thus wound healing dynamics is a nonlinear process. The choice and assessment of this mathematical model was based on periodic weekly measurements of 226 chronic wounds of various aetiologies and treatments. An example of such wound healing dynamics is presented in Fig. 1. A mathematical description of the delayed exponential model is given by eqn 5 .

$$
\hat{S}(t)= \begin{cases}S_{D E X} ; & 0 \leqslant t<T_{D E X} \\ S_{D E X} e^{-\theta_{D E X}\left(t-T_{D E X}\right)} ; & t \geqslant T_{D E X}\end{cases}
$$

where $\hat{S}(t)$ is the estimated wound area in percentage of initial wound area, and three parameters $S_{D E X}, \theta_{D E X}$ and $T_{D E X}$ describe the wound healing dynamics. Parameter $S_{D E X}[\%]$ estimates initial wound area, parameter $\theta_{D E X}\left[\right.$ day $\left.^{-1}\right]$ defines the time constant of the exponent function, and the time delay of the healing process is defined by parameter $T_{D E X}$ [day]. It has been shown that this model has good predictive capability and in this capacity could be used to predict the time needed to complete wound closure. The model may be very useful in clinical trials, where not all wounds included in the study close within the designated study period.

A mathematical model has also been derived to predict the advance of the wound margin towards the wound centre based on the delayed exponential behaviour of the wound area over time. The detailed procedure is explained in Appendix 1. The resulting three-parameter mathematical model is given by

$\hat{d}(t)= \begin{cases}2 \frac{S_{0}}{p_{0}}\left(1-\sqrt{\frac{S_{E R M}}{100}}\right) ; & 0 \leqslant t<T_{E R M} \\ 2 \frac{S_{0}}{p_{0}}\left(1-\sqrt{\frac{S_{E R M}}{100}} e^{\frac{\theta_{E R M}}{2}\left(t-T_{E R M}\right)}\right) ; & t \geqslant T_{E R M}\end{cases}$

where $\hat{d}(t)$ is the estimated advance of the wound margin towards the wound centre, $S_{0}$ is the initial wound area, $p_{0}$ is the initial wound perimeter and $2 S_{0} / p_{0}$ is the maximum advance of the wound margin towards the wound centre, which is, in circular wounds, equal to the circle radius millimetres. Parameter $S_{E R M}[\%]$ estimates the initial advance of the wound margin towards the wound centre. Parameter $\theta_{E R M}\left[\right.$ day $\left.^{-1}\right]$ defines the time constant of the 


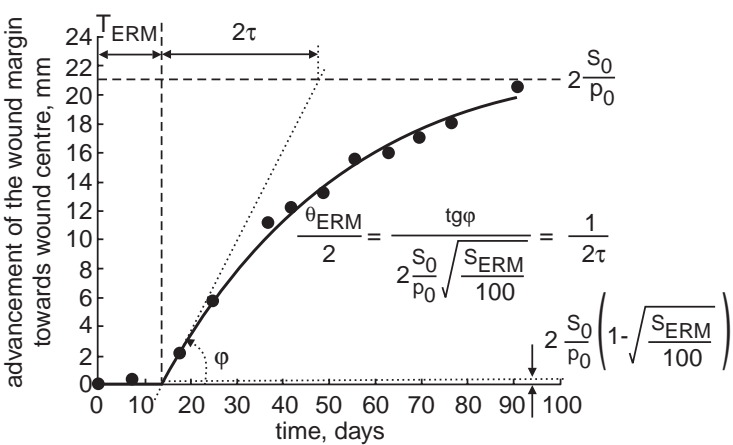

Fig. 2 Example of measuring wound area and perimeter over time, calculating advancement of the wound margin towards wound centre and applying delayed exponential rise to maximum model to calculated data. $S_{E R M}, \theta_{E R M}$ and $T_{E R M}$ are the calculated parameters of the fitted delayed exponential rise to maximum model. ( $\rightarrow$ Fitted delayed exponential rise to maximum model; (•) advancement of wound margin towards wound centre

exponent function. Positive values relate to healing wounds and negative values to nonhealing wounds. The time delay of the healing process initiation is defined by parameter $T_{E R M}$ [day]. An example of the advance of the wound margin towards the wound centre is presented in Fig. 2 for the same wound shown in Fig. 1.

In the proliferative phase of healing as collagen accumulates, the wound contracts allowing the wound margin to move towards the wound centre (BARDSLEY et al., 1995).

We also considered approximating wound shape by a circle, eliminating any information about the actual wound shape. When the dynamics of the advance of the wound margin towards the wound centre was calculated based on a circular wound shape approximation, the maximum advance of the wound margin towards the wound centre was overestimated. Relative overestimation expressed as a function of width to length ratio is presented in eqn 23 (Appendix 2).

\section{Wound healing rate definition}

Previously it was proved that the healing dynamic is nonlinear regardless of how the wound extent is measured. Since wound healing is often delayed, the measurement function cannot be linearised. The majority of authors have used a measure of the wound healing rate that assumes linear behaviour over time. This assumption is misleading and definitions of wound healing rate based only on two measurements of wound extent performed during the study period do not accurately reflect dynamic changes in wound extent.

The goal of wound care is complete wound closure. Therefore, wound healing rate should describe the time needed to wound closure, directly or indirectly. Because clinical trials are limited by time and financial support, not all wounds are followed regularly or until complete wound closure. In these cases the investigator should be able to mathematically predict the wound closure time. Prediction based on wound healing rate calculated from the initial wound extent measurement and one measurement obtained during wound healing is inaccurate because it does not take into account that wound healing dynamics are nonlinear. To accurately reflect changes in the wound it has to be measured periodically and a known model of the healing dynamics fitted to the obtained data. From calculated values of model parameters the time needed to complete wound closure can be predicted. Because the exponential function does not reach its asymptote until infinite time, the wound was defined to be closed when the mathematically predicted wound area is smaller than $5 \%$ of the initial value and at the same time smaller than $100 \mathrm{~mm}^{2}$. In Appendix 3 the mathematical expression for prediction of the time needed to complete wound closure $T$ (eqn 26) is defined according to these two requirements.

Wound healing rates defined as absolute area healed per day (eqn 7), as percentage of initial area healed per day (eqn 8) and as advance of the wound margin towards the wound centre per day (eqn 9), were compared:

$$
\begin{aligned}
& \Theta_{\text {absolute }}=\frac{S_{0}}{T}\left[\mathrm{~mm}^{2} / \text { day }\right] \\
& \Theta_{\text {relative }}=\frac{100}{T}[\% / \text { day }] \\
& \Theta_{\text {edge }}=2 \frac{S_{0}}{p_{0} T}[\mathrm{~mm} / \text { day }]
\end{aligned}
$$

Wound healing rate should not be affected by wound size when wounds of different sizes are compared. To examine the effect of wound area, perimeter and width to length ratio on these three wound healing rates, correlation analysis was performed on the clinical data.

\section{Testing on clinical data}

Two mutually perpendicular diameters of 300 chronic wounds were measured periodically every week: in total 2481 measurements were performed. Wound area and perimeter were calculated according to eqns 2 and 4, respectively. Because wound area is a function of the width to length ratio and wound perimeter is a function of the square root of this ratio, wound perimeter was squared. The squared perimeter was then compared to the surface and ratio. Initial wound area $S_{0}$ was strongly correlated $(r=0.990, n=300)$ ( $r=$ Pearson correlation coefficient, $n=$ number of cases) with the squared initial wound perimeter $p_{0}$, while initial width to length ratio $r_{0}$ was mildly correlated with the initial wound area $(r=0.201, n=300)$, and not correlated to the squared initial perimeter $(r=0.116$, $n=300)$. Considering all wound measurements it was found that wound area was strongly correlated $(r=0.985, n=2481)$ with the squared wound perimeter, while width to length ratio was modestly correlated with wound area $(r=0.211, n=2481)$, and not to squared perimeter $(r=0.147, n=2481)$. It seems that wound area is more linearly dependent on wound shape than perimeter, although both correlations are negligible. A strong positive correlation between and area was anticipated.

Average, minimal and maximal width to length ratio $r_{i}$ over the observation time for each of 300 wound cases was calculated. The mean of the average values with standard deviation is $0.663 \pm 0.170(n=300)$, while the mean of the minimal values with standard deviation is $0.476 \pm 0.206(n=300)$, and the mean of the maximal values with standard deviation is $0.899 \pm 0.167$ $(n=300)$. Maximal values are close to one, since a closed wound (last measurement) was considered to have width to length ratio equal to one. Mean initial width to length ratio with standard deviation is $0.672 \pm 0.216(n=300)$. Comparing the initial and average width to length ratio with paired $t$-test showed no significant difference $(p=0.260)$. Therefore, it was concluded that width to length ratio does not change significantly during the healing process. Mean width to length ratio of all measurements performed in the study with standard deviation was $0.653 \pm 0.229(n=2481)$. GORIN et al. (1996) reported a mean initial width to length ratio $0.58 \pm 0.20(n=49)$, which is lower, but still close to current findings $(p=0.027)$. 
Relative overestimation of the maximum advance of the wound margin towards the wound centre of the circular approximation (eqn 23) is a function of width to length ratio. Assuming that the ratio does not change over time, overestimation at a mean initial width to length ratio 0.672 is only $3.0 \%$.

\subsection{Wound healing dynamics}

In general, wounds are of an irregular shape. To facilitate wound size assessment wound shape is approximated either with an ellipse or a circle. A statistical analysis of clinical data was performed to determine if there is any difference in the parameters $S_{E R M}, \theta_{E R M}$ and $T_{E R M}$ of wound healing dynamics when wound shape is approximated with an ellipse or a circle. For the elliptical approximation of each wound, advance of the wound margin towards the wound centre, values $d_{i}, i=0,1, \ldots, n-1$, were calculated from $n$ periodically measured wound diameters. The model termed the delayed exponential rise to maximum model (eqn 6) was fitted to the calculated values of $d_{i}$.

For the circular wound shape approximation wound diameters were recalculated to attain a width to length ratio 1.0 without changing the wound area. From recalculated diameters values of $d_{i}, i=0,1, \ldots, n-1$, were calculated and the 'delayed exponential rise to maximum model' fitted to these values.

For each wound case comparisons were made of the parameters of the 'delayed exponential rise to maximum model' calculated with elliptical and circular wound shape approximation and the parameters of the 'delayed exponential model'. From the results presented in Table 2, it is evident that wound shape does not significantly affect model parameters. The measure 'advance of the wound margin towards the wound centre' is dependent on wound shape, but the effect of wound shape on wound healing dynamics is not significant. Since it is easier to measure wound area than wound area and perimeter, wound healing dynamics was accurately estimated from regular

Table 2 Paired sample t-tests performed on each parameter calculated from the advance of the wound margin towards the wound centre model (elliptical and circular approximation) and wound area dynamics model. Calculating natural logarithm normalised nonnormal distribution of parameter $\theta$. Bonferroni adjustment probability was used

\begin{tabular}{lccc}
\hline & $S_{D E R \text { ellipse }}$ & $S_{D E R \text { circle }}$ & $S_{D E X}$ \\
\hline$S_{D E R \text { ellipse }}$ & 1.000 & 1.000 & 0.026 \\
$S_{D E R \text { circle }}$ & & 1.000 & 0.083 \\
$S_{D E X}$ & & & 1.000 \\
\hline & $\theta_{D E R \text { ellipse }}$ & $\theta_{D E R \text { circle }}$ & $\theta_{D E X}$ \\
\hline$\theta_{D E R \text { ellipse }}$ & 1.000 & 0.275 & 0.052 \\
$\theta_{D E R \text { circle }}$ & & 1.000 & 0.236 \\
$\theta_{D E X}$ & & & 1.000 \\
\hline & $T_{D E R}$ ellipse & $T_{D E R \text { circle }}$ & $T_{D E X}$ \\
\hline$T_{D E R}$ ellipse & 1.000 & 0.128 & 0.159 \\
$T_{D E R \text { circle }}$ & & 1.000 & 0.371 \\
$T_{D E X}$ & & & 1.000 \\
\hline
\end{tabular}

Table 3 Mean values with standard deviations of parameters describing healing dynamics of 300 wound cases and their median values with interquartile range

\begin{tabular}{lllc}
\hline & \multicolumn{1}{c}{$S_{D E X}$} & \multicolumn{1}{c}{$\theta_{D E X}$} & $T_{D E X}$ \\
\hline Mean & 98.1 & 0.068 & 8.8 \\
Standard deviation & 11.9 & 0.079 & 14.4 \\
Median & 99.8 & 0.049 & 3.6 \\
Interquartile range & $95.9-100.0$ & $0.020-0.091$ & $0.0-11.1$ \\
\hline
\end{tabular}

wound area measurements (eqn 5). In Table 3 mean parameter values of the delayed exponential model for 300 wound cases are presented. Out of 300 wound cases only 174 were closed during the experimental period. For this group, time needed to complete wound closure was calculated using the mathematical model and compared to the actual time needed to complete wound closure. Paired sample $t$-tests were performed on the calculated time needed to complete wound closure versus the actual time needed to complete wound closure in 174 wound cases (distribution of time needed to complete wound closure was normal). This resulted in a probability 0.958 . The difference was insignificant, which means that the model and the criteria of wound closure estimate time needed to complete wound closure accurately.

From 174 wound cases that were closed during the study period, wound cases were selected that were assessed weekly with no missing measurements. The delayed exponential model was applied to wound area measurements assessed during the first three to six weeks. The time needed to complete wound closure was predicted from parameters of the delayed exponential model for each wound case. Predicted times were compared to the actual times wounds needed to close. Because distribution of the time needed to complete wound closure was nonnormal, data were transformed using natural logarithms to achieve normal distribution. Results of paired $t$-tests are presented in Table 4. The difference between the actual time needed to complete wound closure and the time predicted from measurements of wound area in the first three weeks of the observation period (four measurements) was significant. No statistically significant difference was found between the actual time to complete wound closure and the time predicted from measurements of wound area in the first four or more weeks of observation period (five or more measurements). Therefore, the wound should be observed for at least four weeks to allow time to predict complete wound closure.

\subsection{Wound healing rate}

Pearson correlation analysis $(r=$ Pearson correlation coefficient, $p=$ probability of being wrong in concluding that there is a true association between the variables) was used to examine the effect of wound area, perimeter (they were logged to achieve normal distribution) and width to length ratio on the calculated wound healing rates (for 300 wound cases) according to eqns 7-9. When healing rate was calculated as absolute area healed per day, healing rates correlated with the initial wound area ( $r=0.580, p<0.001)$, perimeter $(r=0.571, p<0.001)$ but not with width to length ratio $(r=0.132, p=0.170)$. When healing rate was expressed as a percentage of initial area healed per day, healing rates moderately correlated with initial wound area $(r=-0.431, p<0.001)$ and perimeter $(r=-0.413, p<0.001)$ but not with width to length ratio $(r=-0.153, p=0.067)$. When healing rate was expressed as the advance of the wound margin towards the wound centre per day, no correlation with initial

Table 4 Results ( $p$ values) of paired t-test between actual time needed to complete wound closure and predicted time needed to complete wound closure after three to six weeks of observation. During the observation period 174 wounds were closed. They were closed in $8.4 \pm 5.6$ (mean $\pm S D$ ) weeks, mininum 3 and maximum 34 weeks, with median (interquartile range) 6 (5-11) weeks. Before analysis was performed, values were transformed using the normal logarithm

\begin{tabular}{lc}
\hline Predicted healing time & Actual time \\
\hline After 3 weeks & $p<0.001$ \\
After 4 weeks & $p=0.062$ \\
After 5 weeks & $p=0.484$ \\
After 6 weeks & $p=0.900$ \\
\hline
\end{tabular}


wound area $(r=0.146, p=0.091)$, perimeter $(r=0.121$, $p=0.266)$, or width to length ratio $(r=0.105, p=0.493)$ was found. Wound healing rate expressed as absolute area healed per day, tends to exaggerate the healing rates of larger wounds, and healing rate expressed as a percentage of initial area healed per day, tends to exaggerate the healing rates of smaller wounds. Only wound healing rate expressed as the advance of the wound margin towards the wound centre per day, is not influenced by initial wound size.

The median initial wound area of 300 wound cases was $633 \mathrm{~mm}^{2}$. When all the wounds in this study were divided into a group of smaller $\left(S_{0}<633 \mathrm{~mm}^{2}\right)$ and larger wounds $\left(S_{0}>633 \mathrm{~mm}^{2}\right)$ no significant difference $(p=0.338)$ was found in mean values of healing rate expressed as the advance of the wound margin towards the wound centre per day using a two-sample $t$-test. The differences in mean values of healing rate expressed as the absolute area healed per day and in mean values of healing rate expressed as the percentage of initial area healed per day between small and large group of wounds were significant $(p<0.001)$. Therefore, it is proposed that wound healing rate expressed as the advance of the wound margin towards the wound centre per day be used when healing rates of wounds with different initial areas are compared.

\section{Conclusion}

One of the most important principles of chronic wound management is periodic assessment of wound healing. It is important to document healing progress, and to assess the effectiveness of treatment so as to maximise healing rates through treatment optimisation. A number of measures of wound healing rate have been proposed and used. None of them is firmly established for either clinical or research purposes. Different wound status assessment techniques and different wound healing rate definitions render published reports (Table 1) difficult to compare. In this paper different techniques of wound healing rate evaluation were compared and it is proposed that wound healing rate should be defined as the advance of the wound margin towards the wound centre per day. Many trial studies are financially and time restricted and thus observation time is limited. Clinicians need a reliable method of predicting wound closure so that they can provide this information to insurance companies for reimbursement. Since the wound healing process is nonlinear and cannot be made linear by any transformation, only a nonlinear model can be used to describe wound healing progress. Linear estimation of healing progress is incorrect and can lead to deceptive results. It has been shown on 300 electrically stimulated or conventionally treated chronic wound cases that wound healing dynamics is not influenced by wound shape, which indicates that wound area measurements are sufficient for its adequate description. A delayed exponential model was fitted to normalised wound area measurements (percentage of initial wound area). In this regard, wound area should be periodically assessed for at least four weeks with a minimum of five wound area or mutually perpendicular diameter measurements recorded over this time. The time needed to complete wound closure is calculated from delayed exponential model parameters.

Only a generally accepted uniform wound healing rate definition would enable comparison of treatment efficacy by different research groups. The proposed measure of wound healing rate is simple to use if wounds are closed within the observation time period. When model fitting is required, the proposed method requires use of a computer with appropriate software. This is the most adequate estimation of wound healing rate although its complexity could be a drawback because a personal computer is needed. However, computers and informatics are now common in clinics and this drawback will soon be of no consequence.

In further work we are planning to set an application for wound healing rate prediction on the Web.

Acknowledgments-This research was supported by the Ministry of Science and Technology of the Republic of Slovenia. The authors wish to express their appreciation to Dr Renata Karba for helpful suggestions and comments during the preparation of the manuscript.

\section{Appendix 1}

This appendix discusses the time dependence of the advance of the wound margin towards the wound centre. The advance of the wound margin towards the wound centre $\left(d_{i}\right)$ is defined as an absolute change of wound area $\left(\Delta S_{i}\right)$ divided by mean wound perimeter $\left(\bar{p}_{i}\right)$ in the time interval $t_{0}$ to $t_{i}$ (eqn 1). Wound shape can be approximated with an ellipse and wound area $\left(S_{i}\right)$ calculated from two mutually perpendicular diameters $\left(a_{i}\right.$ and $b_{i}$ ) of the wound taken at time $t_{i}$ (eqn 2). The two diameters define width to length ratio $r_{i}$ (eqn 3 ). As ratio $r_{i}$ and wound area $S_{i}$ change over time, perimeter $p_{i}$ (eqn 4 ) also changes over time. Mean wound perimeter $\bar{p}_{i}$ was calculated for each periodical assessment $i=0,1, \ldots, n-1$ :

$$
\begin{aligned}
\bar{p}_{i} & =\frac{p_{0}+p_{i}}{2} \\
& =\frac{\pi}{4}\left[\frac{3}{2}\left(a_{0}+b_{0}+a_{i}+b_{i}\right)-\sqrt{a_{0} b_{0}}-\sqrt{a_{i} b_{i}}\right]
\end{aligned}
$$

Wound area has a delayed exponential behaviour over time (CUKJATi et al., 2000):

$$
\hat{S}_{D E X_{i}}= \begin{cases}S_{D E X} ; & 0 \leqslant t_{i}<T_{D E X}[\%] \\ S_{D E X} e^{-\theta_{D E X}\left(t_{i}-T_{D E X}\right)} ; & t_{i} \geqslant T_{D E X}\end{cases}
$$

where $T_{D E X} \geq 0$. Nonlinear regression of eqn 11 to $n$ normalised wound area values measured over time results in parameters $S_{D E X}, \theta_{D E X}$ and $T_{D E X}$, which describe wound healing progress for the specific wound case. $\hat{S}_{D E X_{i}}$ is the estimated wound area at time $t_{i}$ as a percentage of initial wound area. It was multiplied by the absolute initial wound area $S_{0}\left(\mathrm{~mm}^{2}\right)$ and divided by 100 to achieve an absolute value of wound area $\hat{S}_{i}$ :

$$
\begin{aligned}
\hat{S}_{i} & =S_{0} \frac{\hat{S}_{D E X i}}{100} \\
& = \begin{cases}S_{0} \frac{S_{D E X}}{100} ; & 0 \leqslant t_{i}<T_{D E X} \\
S_{0} \frac{S_{D E X}}{100} e^{-\theta_{D E X}\left(t_{i}-T_{D E X}\right)} ; & \left.t_{i} \geqslant \mathrm{~mm}^{2}\right]\end{cases}
\end{aligned}
$$

where $S_{0} S_{D E X} / 100$ is the estimated initial wound area $\left(\mathrm{mm}^{2}\right)$.

Considering eqn 12, absolute change in wound area at time $t_{i}$ was calculated according to

$$
\begin{aligned}
\Delta \hat{S}_{i}= & S_{0}-\hat{S}_{i} \\
= & \begin{cases}S_{0}-S_{0} \frac{S_{D E X}}{100} e^{-\theta_{D E X}\left(t_{i}-T_{D E X}\right)} ; & t_{i} \geqslant T_{D E X} \\
S_{0}\left(1-\frac{S_{D E X}}{100}\right) ; & 0 \leqslant t_{i}<T_{D E X}\end{cases}
\end{aligned}
$$


Determination of healing dynamics was performed in two steps. In the first step mean wound perimeter $\bar{p}_{i}$ was determined in terms of ratio $r_{i}$ and estimated wound area $\hat{S}_{D E X i}$. In the second step the advance of the wound margin towards the wound centre was obtained over time.

Considering eqns 2 and 3 , we can express ellipse axes $a_{i}$ and $b_{i}$ as terms of ratio $r_{i}$ and estimated wound area $S_{i}$ :

$$
\begin{aligned}
& \left.\begin{array}{rl}
a_{i} & =\frac{4}{\pi} \frac{S_{i}}{b_{i}} \\
b_{i} & =r_{i} \cdot a_{i}
\end{array}\right\} \Longrightarrow a_{i}=\sqrt{\frac{4}{\pi} S_{i} \frac{1}{r_{i}}} \\
& \left.\begin{array}{l}
b_{i}=\frac{4}{\pi} \frac{S_{i}}{a_{i}} \\
a_{i}=\frac{1}{r_{i}} \cdot b_{i}
\end{array}\right\} \Longrightarrow b_{i}=\sqrt{\frac{4}{\pi} S_{i} \cdot r_{i}}
\end{aligned}
$$

After inserting eqn 14 and eqn 15 in eqn 10 , mean perimeter is expressed in terms of ratio $r_{i}$ and wound area $S_{i}$ :

$$
\begin{aligned}
\bar{p}_{i}= & \frac{\pi}{4}\left[\frac { 3 } { 2 } \left(\sqrt{\frac{4}{\pi} S_{0} \frac{1}{r_{0}}}+\sqrt{\frac{4}{\pi} S_{0} r_{0}}\right.\right. \\
& \left.\left.+\sqrt{\frac{4}{\pi} S_{i} \frac{1}{r_{i}}}+\sqrt{\frac{4}{\pi} S_{i} r_{i}}\right)-\sqrt{\frac{4}{\pi} S_{0}}-\sqrt{\frac{4}{\pi} S_{i}}\right] \\
\bar{p}_{i} & =k_{0} \sqrt{S_{0}}+k_{i} \sqrt{S_{i}}
\end{aligned}
$$

where

$$
k_{i}=\sqrt{\frac{\pi}{4}}\left[\frac{3}{2}\left(\sqrt{\frac{1}{r_{i}}}+\sqrt{r_{i}}\right)-1\right]
$$

Replacing wound area $S_{i}$ in eqn 16 with estimated wound area $\hat{S}_{i}$ (eqn 12) results in the estimated mean wound perimeter $\overline{\hat{p}}_{i}$ :

$$
\begin{aligned}
& \overline{\hat{p}}_{i}=k_{0} \sqrt{S_{0}}+k_{i} \sqrt{\hat{S}_{i}} \\
& \overline{\hat{p}}_{i}= \begin{cases}\sqrt{S_{0}}\left(k_{0}+k_{i} \sqrt{\frac{S_{D E X}}{100}}\right) ; & 0 \leqslant t_{i}<T_{D E X} \\
\sqrt{S_{0}}\left(k_{0}+k_{i} \sqrt{\frac{S_{D E X}}{100}} e^{\frac{-\theta_{D E X}}{100}\left(t_{i}-T_{D E X}\right)}\right) ; & t_{i} \geqslant T_{D E X}\end{cases}
\end{aligned}
$$

By dividing eqn 13 by eqn 17 we obtain a function of time that describes the dynamics of the advance of the wound margin towards the wound centre.

$$
\begin{aligned}
\hat{d}_{i}= & \frac{\Delta \hat{S}_{i}}{\hat{\hat{p}}_{i}} \\
& = \begin{cases}\sqrt{S_{0}} \frac{1-\frac{S_{D E X}}{100}}{k_{0}+k_{i} \sqrt{\frac{S_{D E X}}{100}} ;} & 0 \leqslant t_{i}<T_{D E X} \\
\sqrt{S_{0}} \frac{1-\frac{S_{D E X}}{100} e^{-\theta_{D E X}\left(t_{i}-T_{D E X}\right)}}{k_{0}+\sqrt{\frac{S_{D E X}}{100}} k_{i} e^{\frac{-\theta_{D E X}}{2}\left(t_{i}-T_{D E X}\right)}} ; & t_{i} \geqslant T_{D E X}\end{cases}
\end{aligned}
$$

If we assume that width to length ratio $r_{i}$ does not change during the wound healing process, $k_{i}=k_{0}, i=0,1, \ldots, n$, then eqn 18 reduces to

$$
\hat{d}_{i}= \begin{cases}\frac{\sqrt{S_{0}}}{k_{0}} \frac{1-\frac{S_{D E X}}{100}}{1+\sqrt{\frac{S_{D E X}}{100}}} ; & 0 \leqslant t_{i}<T_{D E X} \\ \frac{\sqrt{S_{0}}}{k_{0}} \frac{1-\frac{S_{D E X}}{100} e^{-\theta_{D E X}\left(t_{i}-T_{D E X}\right)}}{1+\sqrt{\frac{S_{D E X}}{100}} e^{\frac{-\theta_{D E X}}{2}\left(t_{i}-T_{D E X}\right)}} ; & t_{i} \geqslant T_{D E X}\end{cases}
$$

After considering

$$
\begin{aligned}
& (1-x) /(1+\sqrt{x})=1-\sqrt{x}, \\
& \frac{\sqrt{S_{0}}}{k_{0}}=2 \frac{S_{0}}{p_{0}}
\end{aligned}
$$

and re-indexing parameters ( $D E X$ is renamed $E R M)$ the final equation is obtained:

$$
\hat{d}_{i}= \begin{cases}2 \frac{S_{0}}{p_{0}}\left(1-\sqrt{\frac{S_{E R M}}{100}}\right) ; & 0 \leqslant t_{i}<T_{E R M} \\ 2 \frac{S_{0}}{p_{0}}\left(1-\sqrt{\left.\frac{S_{E R M}}{100} e^{\frac{-\theta_{E R M}}{2}\left(t_{i}-T_{E R M}\right)}\right) ;}\right. & t_{i} \geqslant T_{E R M}\end{cases}
$$

It can be shown that $\hat{d}_{i}$ follows a 'delayed exponential rise to maximum' behaviour, which can be mathematically expressed as a function of three parameters $S_{E R M}, \theta_{E R M}$ and $T_{E R M}$, where $T_{E R M} \geqslant 0$.

\section{Appendix 2}

Wound shape can also be approximated by a circle containing the actual wound area. However, in this case all information about actual wound shape is lost. Circular approximation of wound shape is just a special case of elliptical approximation, with width to length ratio equal to one and not changing over time. Considering the perimeter of the circle

$$
p_{0}=2 \sqrt{\pi S_{0}}
$$

in eqn 20 results in the circular wound shape approximation based model of the advance of the wound margin towards the wound centre dynamics:

$$
\hat{d}(t)= \begin{cases}\sqrt{\frac{S_{0}}{\pi}}\left(1-\sqrt{\frac{S_{E R M}}{100}}\right) ; & 0 \leqslant t<T_{E R M} \\ \sqrt{\frac{S_{0}}{\pi}}\left(1-\sqrt{\frac{S_{E R M}}{100}} e^{\frac{-\theta_{E R M}}{2}\left(t_{i}-T_{E R M}\right)}\right) ; \quad t \geqslant T_{E R M}\end{cases}
$$

Parameters $S_{E R M}, \theta_{E R M}$ and $T_{E R M}$ are the same for both elliptical (eqn 20) and circular (eqn 21) wound shape approximation based models of wound healing dynamics. However, when wounds of noncircular shape are approximated by a circle, the maximum advance of the wound margin towards the wound centre $S_{0} / p_{0}$ is overestimated, because a circle has the lowest perimeter for a given area. The relative difference between the circular and exponential approximation (eqn 22) is calculated by subtracting eqn 21 from eqn 20 and dividing by eqn 20 .

$$
e_{0}=\frac{\sqrt{\frac{S_{0}}{\pi}}-2 \frac{S_{0}}{p_{0}}}{2 \frac{S_{0}}{p_{0}}} \cdot 100=\left[\frac{p_{0}}{2 \sqrt{\pi S_{0}}}-1\right] \cdot 100
$$


The relative difference expressed in terms of initial width to length ratio is given by

$$
e_{0}=\frac{3}{2}\left[\frac{1}{2}\left(\sqrt{\frac{1}{r_{0}}}+\sqrt{r_{0}}\right)-1\right] \cdot 100 \quad[\%]
$$

\section{Appendix 3}

As the final goal of wound treatment is closure then the wound healing rate should contain information about the time needed to complete wound closure. If a wound defect is totally resurfaced by epithelium during the experimental period, the time to complete wound closure is known. In wound cases where the wound is not completely closed during the experimental period, time to complete wound closure can be predicted using the delayed exponential model parameters. Because the exponential curve becomes asymptotic at infinity (i.e. the time to complete wound closure is infinite), criteria must be defined to specify when the wound is considered to be closed. The wound was defined to be closed when the mathematically estimated wound area is less than $5 \%$ of the initial wound area and simultaneously less than one square centimetre.

Wound area falls to $5 \%$ of the initial area after $x$ time constants $(\tau)$ (Fig. 2). The exact value of $x$ is given by

$$
\begin{aligned}
& S_{D E X} e^{-\theta_{D E X}\left(t_{i}-T_{D E X}\right)}=5 \mid \ln \\
& -\theta_{D E X} \underbrace{\left(t-T_{D E X}\right)}_{\frac{x}{\theta_{D E X}}}=\ln \frac{5}{S_{D E X}}=-x \Longrightarrow x=\ln \frac{S_{D E X}}{5} \doteq 3 \tau
\end{aligned}
$$

The time needed for the wound area to fall to $5 \%$ of the initial area is $T_{D E X}+x \tau$. Accordingly, the time needed to complete wound closure, $T_{5 \%}$, is given by

$$
T_{5 \%}=T_{D E X}+x \tau=T_{D E X}+\ln \frac{S_{D E X}}{5} \frac{1}{\theta_{D E X}}[\text { day }]
$$

Wound area falls to $100 \mathrm{~mm}^{2}$ after $y$ time constants $(\tau)$ :

$$
\begin{aligned}
\frac{S_{D E X}}{100} S_{0} e^{-\theta_{D E X}\left(t-T_{D E X}\right)} & =100 \mid \ln \\
-\theta_{D E X} \underbrace{\left(t-T_{D E X}\right)}_{\frac{y}{\theta_{D E X}}} & =\ln \left(\frac{100}{S_{0}} \frac{100}{S_{D E X}}\right) \\
& =-y \Rightarrow y=\ln \left(\frac{S_{0} S_{D E X}}{10^{4}}\right)
\end{aligned}
$$

The time needed for the wound area to fall to $100 \mathrm{~mm}^{2}$ is $T_{D E X}+y \tau$. Accordingly, the time needed to complete wound closure $T_{100}$ is given by

$$
T_{100}=T_{D E X}+y \tau=T_{D E X}+\ln \left(\frac{S_{0} S_{D E X}}{10^{4}}\right) \frac{1}{\theta_{D E X}}[\text { day }]
$$

The time needed to complete wound closure $T$ is the greater of $T_{5 \%}$ and $T_{100}$. If wound is not healing, time is negative, describing time needed to double wound area.

$$
T= \begin{cases}T_{5 \%} ; & \text { if }\left|T_{5 \%}\right| \geqslant\left|T_{100}\right| \\ T_{100} ; & \text { otherwise }\end{cases}
$$

\section{References}

BAKER, L. L., ChAMBERs, R., DeMuth, S. K., and Villar, F. (1997): 'Effects of electrical stimulation on wound healing in patients with diabetic ulcers', Diabetes Care, 20, pp. 405-412
Bardsley, W. G., SATtar, A., Armstrong, J. R., Shah, M., Brosnan, P., and Ferguson, M. W. J. (1995): 'Quantitative analysis of wound healing', Wound Rep. Reg., 3, pp. 426-441

BARTOLUCCI, A. A., and ThOMAS, D. R. (1997): 'Using principal component analysis to describe wound status', Adv. Wound Care, 10, pp. 93-95

Birke, J. A., Novick, A., Patout, C. A., and Coleman, W. C. (1992): 'Healing rates of plantar ulcers in leprosy and diabetes', Leprosy Rev., 63, pp. 365-374

Brem, H., BAlleduX, J., IoOM, T., Kerstein, M. D., and Hollier, L. (2000): 'Healing of diabetic foot ulcers and pressure ulcers with human skin equivalent: a new paradigm in wound healing', Arch. Surg., 135, pp. 627-634

Brown, M. (1995): 'Ultrasound for wound management', in GoGIA, P. P. (Ed): 'Clinical wound management' (SLACK Incorporated, Thorofare), pp. 197-206

Covington, J. S., Griffin, J. W., Mendius, R. K., Tooms, R. E., and CLIFFT, J. K. (1989): 'Measurement of pressure ulcer volumes using dental impression materials: suggestions from the field', Phys. Ther., 69, pp. 690-694

CudDigan, J. (1997): 'Pressure ulcer classification: What do we have? What do we need?', Adv. Wound Care, 10, pp. 13-15

CukJati, D., ReberšeK, S., KARBA, R., and MiklavČič, D. (1998): 'Mathematical modeling of chronic wound healing', Electro- Magnetobiol., 17, pp. 235-240

CUKJATI, D., KARBA, R., REBERŠEK, S., and MiKLAVČIČ, D. (2000): 'Modeling of chronic wound healing dynamics', Med. Biol. Eng. Comput., 38, pp. 339-347

Feedar, J. A., Kloth, L. C., and Gentzkow, G. D. (1991): 'Chronic dermal ulcer healing enhanced with monophasic pulsed electrical stimulation', Phys. Ther., 71, pp. 639-649

Ferrell, B. A., Artinian, B. M., and Sessing, D. (1995): 'The sessing scale for assessment of pressure ulcer healing', J. Am. Geriatr. Soc., 43, pp. 37-40

GILMAN, T. H. (1990): 'Parameter for measurement of wound closure', Wounds, 3, pp. 95-101

GoGiA, P. P. (1995a): 'Low-energy laser in wound management', in GoGIA, P. P. (Ed): 'Clinical wound management' (SLACK Incorporated, Thorofare) pp. $165-172$

GogIA, P. P. (1995b): 'Oxygen therapy for wound management', in GoGIA, P. P. (Ed): 'Clinical wound management' (SLACK Incorporated, Thorofare) pp. 185-195

Gorin, D. R., CORDTS, P. R., LAMORTE, W. W., and Menzoian, J. O. (1996): 'The influence of wound geometry on the measurement of wound healing rates in clinical trials', J. Vasc. Surg., 23, pp. 524 528

Jerčinović, A., Karba, R., Vodovnik, L., Stefanovska, A., KrošElJ, P., Turk, R., Džidić, I., BenKo, H., and SAavrin, R. (1994): 'Low frequency pulsed current and pressure ulcer healing', IEEE Trans. Rehab. Eng., 2, pp. 225-233

JOHNSON, M. (1997): 'Using cluster analysis to develop a healing typology in vascular ulcers', J. Vasc. Nurs., 15, pp. 45-49

KANNON, G. A., and GARETT, A. B. (1995): 'Moist wound healing with occlusive dressings. A clinical review', Dermal Surg., 21, pp. 583-590

Karba, R., Šemrov, D., Vodovnik, L., BenKo, H., and Šavrin, R. (1997): 'DC electrical stimulation for chronic wound healing enhancement. Part I. Clinical study and determination of electrical field distribution in the numerical wound model', Bioelectrochem. Bioenergetics, 43, pp. 265-270

KLoth, L. C., and FeEdAR, J. A. (1988): 'Acceleration of wound healing with high voltage, monophasic, pulse current', Phys. Ther, 68, pp. 503-508

KUNIMOTO, B. T. (1999): 'Growth factors in wound healing: the next great innovation?', Ostomy Wound Management, 45, pp. 56-64

LaZArus, G. S., CoOper, D. M., Knighton, D. R., Margolis, D. J., Pecoraro, R. E., Rodeheaver, G., and Robson, M. C. (1994): 'Definitions and guidelines for assessment of wounds and evaluation of healing', Arch. Dermatol., 130, pp. 489-493

LundeberG, T. C. M., ERiKsson, S. V., and Malm, M. (1992): 'Electrical nerve stimulation improves healing of diabetic ulcers', Ann. Plastic Surg., 29, pp. 328-331 
LYMAN, I. R., TENERY, J. H., and BASSON, R. P. (1970): 'Correlation between decrease in bacterial load and rate of wound healing', Surg. Gynecol. Obstet., 130, pp. 616-620

Martin, P., Hopkinson-Woolley, J., and MCCluskey, J. (1992): 'Growth factors and cutaneous wound repair', Prog. Growth Factor Res., 4, pp. 25-44

Nessbaum, E. L., Biemann, I., and Mustard, B. (1994): 'Comparison ultrasound/ultraviolet-C and laser for treatment of pressure ulcers in patients with spinal cord injury', Phys. Ther., 74, pp. 812815

PlassmanN, P., and JONES, B. F. (1992): 'Measuring area and volume of human leg ulcers by colour coded structured light', Wound Care, 1, pp. $35-38$

Singer, A. J., and Clark, R. A. F. (1999): 'Cutaneous wound healing', New England J. Med., 341, pp. 738-745

STEFANOVSKA, A., VODOVNIK, L., BENKO, H., and TURK, R. (1993): 'Treatment of chronic wounds by means of electric and electromagnetic fields. Part 2 Value of FES parameters for pressure sore treatment', Med. Biol. Eng. Comput., 31, pp. 213-220

VODOVNIK, L., and KARBA, R. (1992): 'Treatment of chronic wounds by means or electric and electromagnetic fields. Part 1 Literature review', Med. Biol. Eng. Comput., 30, pp. 257-266
WALDORF, H., and FEWKES, J. (1995): 'Wound healing', Adv. Dermatol., 10, pp. 77-97

Wolcott, L. E., Wheeler, P. C., Hardwicke, H. M., and Rowley, B. A. (1969): 'Accelerated healing of skin ulcers by electrotherapy: preliminary clinical results', South. Med. J., 62, pp. 795-801

\section{Author's biography}

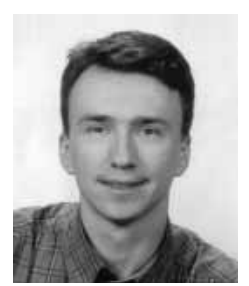

DAVID CUKJATI received his MSc and PhD in Electrical Engineering from the University of Ljubljana. He is presently a Teaching Assistant of Analog, and Digital Electronics and Nonelectrical Control Systems at the Faculty of Electrical Engineering, University of Ljubljana. He works in the field of biomedical engineering. His research interests cover clinical studies of the effects of electric currents on soft tissue healing, database design, modelling, expert systems and the application of classification algorithms to biomedical solutions. 